\title{
Epidemiologic characteristics of haemorrhagic fever with renal syndrome in Mainland China from 2006 to 2010
}

\author{
Xiaoxia Huang, ${ }^{* a}$ Hongmei Yin, ${ }^{* b}$ Lei Yan, ${ }^{a}$ Xiaofang Wang ${ }^{a}$ and Shiwen Wang ${ }^{a}$ \\ Correspondence to Shiwen Wang (e-mail: wangshiwencdc@163.com) and Xiaofang Wang (e-mail: wangxiaofang90@163.com).
}

Objective: To design effective prevention and control strategies for haemorrhagic fever with renal syndrome (HFRS) in Mainland China, we evaluated the epidemiologic characteristics and trends of HFRS cases reported between 2006 and 2010.

Methods: HFRS data from 1970 to 2010 were obtained from the China Notifiable Disease Reporting System (CNDRS). The cases analysed included clinical cases and laboratory-confirmed cases. Data was extracted for statistical analysis by time, region and profession; the incidence rate was obtained directly from CNDRS. In this study, we analysed the morbidity and mortality data of HFRS from 2006 to 2010.

Results: HFRS cases trended downward from 2006 (15 098) to 2009 (8745), but exhibited a slight increase in 2010 (9526). Twenty-nine of 31 provinces reported HFRS cases between 2006 and 2010. Five provinces, namely, Heilongjiang, Jilin and Liaoning in the North-east, Shandong in the east, and Shaanxi in the central part of China, were characterized as high-endemic areas. Seasonal case distribution was bimodal, with peaks of cases in spring and winter. Young male farmers were the most susceptible population to HFRS. Early- to middle-aged adults (20-50 years old) represented the largest groups of HFRS cases.

Conclusion: The overall number of cases of HFRS in China has trended downward possibly due to national vaccine and rodent vector control programmes implemented in the past 25 years. However, this trend slowed down in the last five years. High-endemic regions and at-risk population groups still exist and will benefit from further targeted prevention strategies.

$\mathrm{H}$ aemorrhagic Fever with Renal Syndrome (HFRS), a rodent-borne viral disease caused by different species of Hantaviruses, is characterized by fever, haemorrhagic manifestations and renal dysfunction. ${ }^{1,2}$ In Mainland China, there are two predominant species of Hantavirus, Hantaan and Seoul virus, and either or both species may circulate in a given area. However, studies have revealed that a heterogeneous, gradually evolving, co-circulation of Hantaan and Seoul viruses is most common in Mainland China., ${ }^{3,4}$ This dynamic type of epidemiology is characterized by co-circulation of both viruses with a predominance of Hantaan virus in north-eastern China and a predominance of Seoul virus south-western China.

HFRS is transmitted by contact with rodent urine, feces or saliva. ${ }^{5-7}$ Of the major endemic countries for HFRS, China accounts for $90 \%$ of total HFRS cases worldwide. $^{8}$ The annual reported cases of HFRS surpassed 110000 in $1986 .{ }^{9}$ HFRS cases have been reported in all 31 Chinese provinces in Mainland China.
Although environment management, host surveillance and HFRS vaccine implementation have played an important role in controlling HFRS, it is still a serious disease in Mainland China. ${ }^{2,9-11}$

We analysed data reported from all the provinces of Mainland China between 2006 and 2010 to attain an in-depth understanding of HFRS in recent years. Hopefully, the findings from this study will contribute to the development of more effective HFRS prevention and control strategies.

\section{METHODS}

HFRS is one of the Class B Notifiable Diseases, and data have been reported since 1950 according to a standard protocol. ${ }^{1}$ In this study, HFRS data from 1970 to 2010 was obtained from the China Notifiable Disease Reporting System (CNDRS). ${ }^{1-3}$ The cases analysed included clinical cases and laboratory-confirmed cases. Clinical diagnosis

\footnotetext{
- National Institute for Viral Disease Control and Prevention, Chinese Center for Disease Control and Prevention (China CDC), Beijing, China Chinese Center for Disease Control and Prevention (China CDC), Beijing, China

* Both authors contributed equally in this article

Submitted: 13 May 201 1; Published: 7 February 2012

doi: 10.5365/wpsar.2011.2.2.007
} 
Figure 1. Annual haemorrhagic fever with renal syndrome incidence, China, $1970-2010$

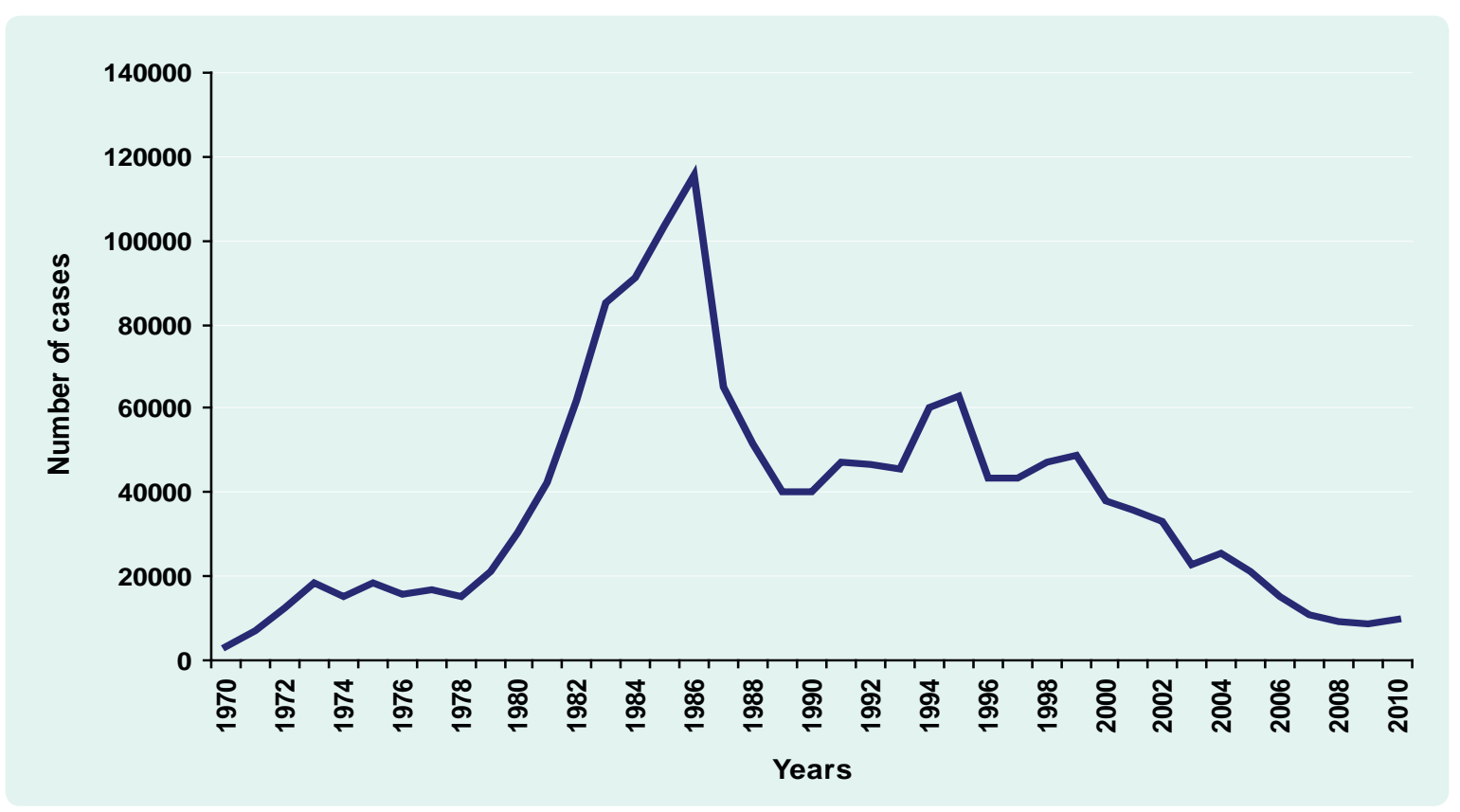

criteria included: exposure history (i.e. direct or indirect exposure to rodents and their excreta and saliva within two months before the onset of illness); acute onset with at least two of the following clinical symptoms (i.e. fever $>38^{\circ} \mathrm{C}$, chills, haemorrhagic manifestations, headache, back pain, abdominal pain, acute renal dysfunction and hypotension); experience or partial experience of the five phases of disease course (i.e. fever, hypopiesis, oliguresis, hyperdiuresis and recovery) and abnormal blood and urine routine parameters. Laboratory-confirmed case diagnosis criteria were clinical diagnosis with one of the positive laboratory tests (HV anti-IgM positive, four-fold increasing of anti-lgG and virus isolated from serum or detected HV RNA). ${ }^{1,12,13}$

All data recorded for HFRS cases from 1970 to 2010 were extracted for statistical analysis by time, region and profession; the incidence rate was obtained directly from CNDRS. We analyzed the morbidity and mortality data of HFRS from 2006 to 2010. This period was China's Eleventh Five-Year Plan, during which time much work was done to prevent and control communicable diseases including HFRS. In 2008, China began the expanded immunization programme for HFRS vaccine, which targeted susceptible people in high-endemic districts to control the HFRS incidence.

Data were organized in Microsoft Excel spreadsheets and processed with SPSS 13.0 software.

\section{RESULTS}

\section{Overview of the HFRS in Mainland China}

From 1970 to 2010, 1546063 HFRS cases were recorded. The annual HFRS incidence rose steadily in the early 1970s but experienced an alarming increase in the early 1980s. Case numbers peaked in 1986, when 115804 cases were reported in Mainland China. From 1987 to 2010, however, HFRS case numbers decreased, with occasional small fluctuations. Eventually, in 2009, HFRS case numbers reached its lowest number (8745) since 1986, followed by a slight rise in 2010 (9526) (Figure 1).

\section{Analysis of HFRS epidemiologic characteristics from 2006 to 2010}

In recent years, the number of HFRS cases had experienced a steady decline. The per cent decline between 2006 and 2009 ranged from $26.73 \%$ (2006 to 2007) to $18.30 \%$ (2007 to 2008) and $3.25 \%$ (2008 to 2009). In 2010, however, the number of reported cases rose. While the number of cases in 2010 (9526 total HFRS cases) was slightly higher than that reported in 2008 (9039) and 2009 (8745), it was still lower than the annual mean number of cases (10 986) between 2006 and 2009. 
Figure 2. Haemorrhagic fever with renal syndrome regional distribution, China, 2006 - 2010

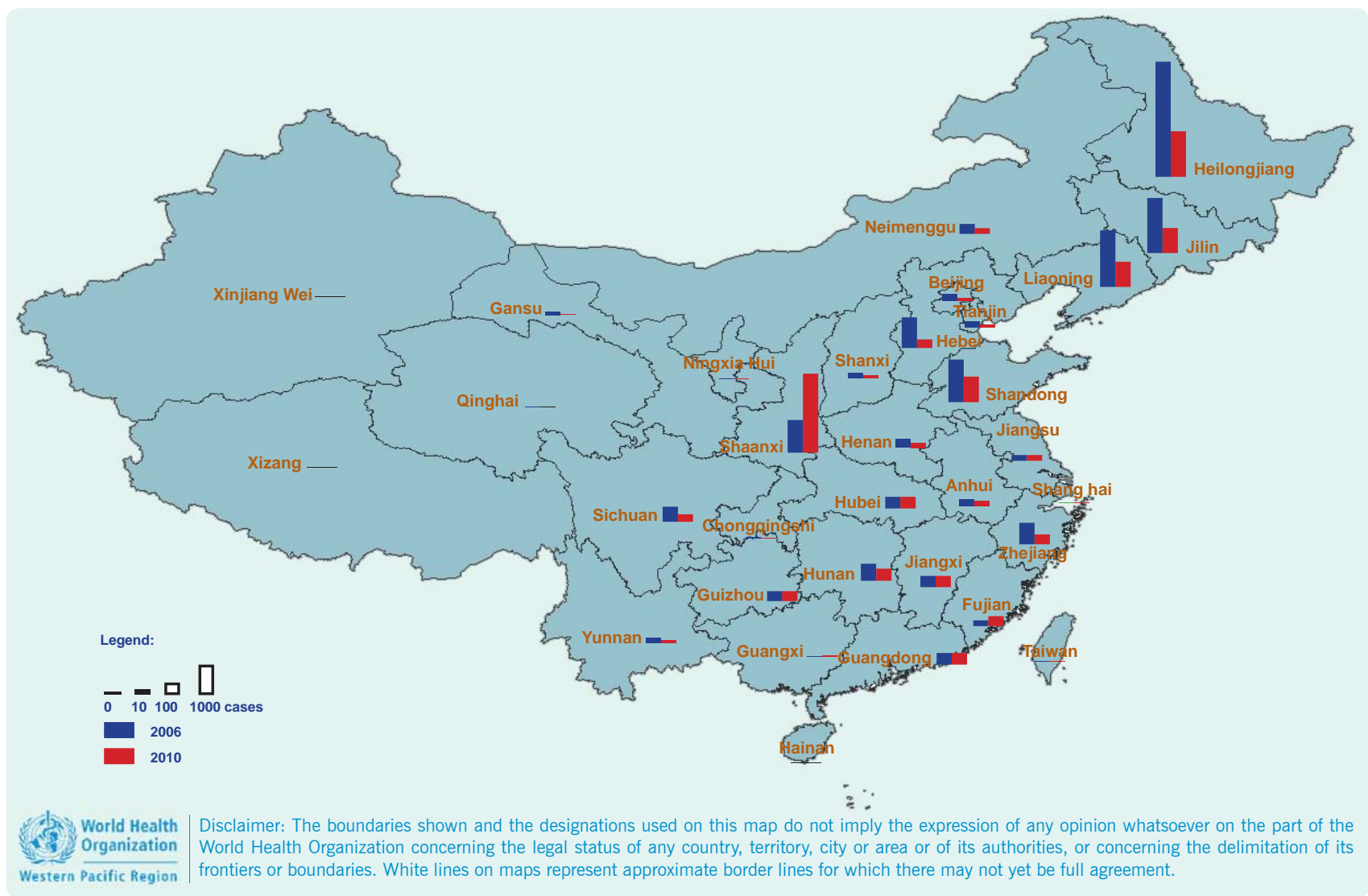

The HFRS incidence rates (per 100000 population) for each year from 2006 to 2010 were $1.15,0.84$, $0.68,0.66$ and 0.71 , while corresponding death numbers and case fatality ratios were $173(1.15 \%)$, 145 (1.31\%), 103 (1.14\%), $104(1.19 \%)$ and 118 $(1.24 \%)$, respectively. The incidence rate in 2010 was determined to be $7.58 \%$ higher than that in 2009 .

From 2006 to 2010, the number of laboratoryconfirmed cases were 5628 (37.28\% of the total cases reported), 3940 (35.61\%), 3202 (35.42\%), 3411 $(39.01 \%)$ and $4830(50.70 \%)$, respectively.

\section{HFRS regional distribution}

From 2006 to 2010, a total of 53471 HFRS cases were reported from 29 provinces. Eight provinces accounted for $80.44 \%$ of cases: Heilongjiang, Shaanxi, Jilin, Shandong, Liaoning, Zhejiang, Hunan and Hebei provinces (listed from highest to lowest number of HFRS cases). Figure 2 shows the HFRS regional distribution in the years 2006 and 2010 and Figure 3 shows the number of cases in the above eight high incidence provinces from 2006 to 2010.

\section{HFRS seasonal distribution}

Two peaks of HFRS cases were observed annually. One peak occurred in the winter and appeared as a relatively high and narrow spike in November. The spring peak was lower and broader, spiking in June. These seasonal distribution profiles were similar for all years between 2006 and 2010, with only a small difference in 2010. In the months before September 2010, number of cases was consistently lower than that of the same period in the years 2006 to 2009. From September onward, however, HFRS case numbers increased, resulting in the highest annual number of cases in all the five years examined (Figure 4).

\section{HFRS population distribution}

From 2006 to $2010,85.60 \%$ of the HFRS cases were represented by the late adolescent-to-adult age group (16-59 years old), ranging from $87.36 \%$ in 2006 to $81.87 \%$ in 2010 (Figure 5). When cases were broken down by 10 year age groups, early- to middle-age (ages 20, 30, 40 and 50) represented the largest groups of HFRS cases. The overall male-to-female ratio of HFRS cases for 2006-2010 was 3.2:1. This distinctive 
Figure 3. Haemorrhagic fever with renal syndrome case numbers in eight high incidence provinces, China, $2006-2010$

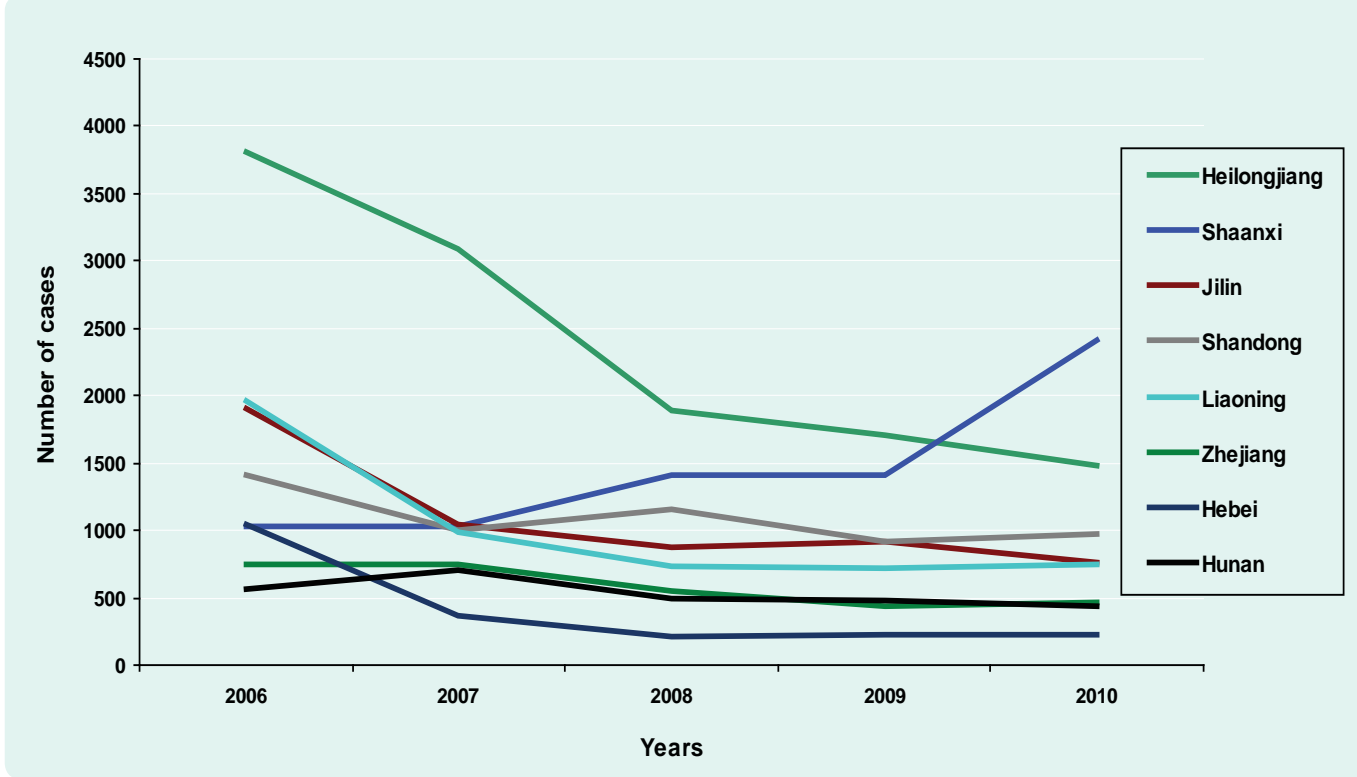

Figure 4. Haemorrhagic fever with renal syndrome seasonal distribution, China, $2006-2010$

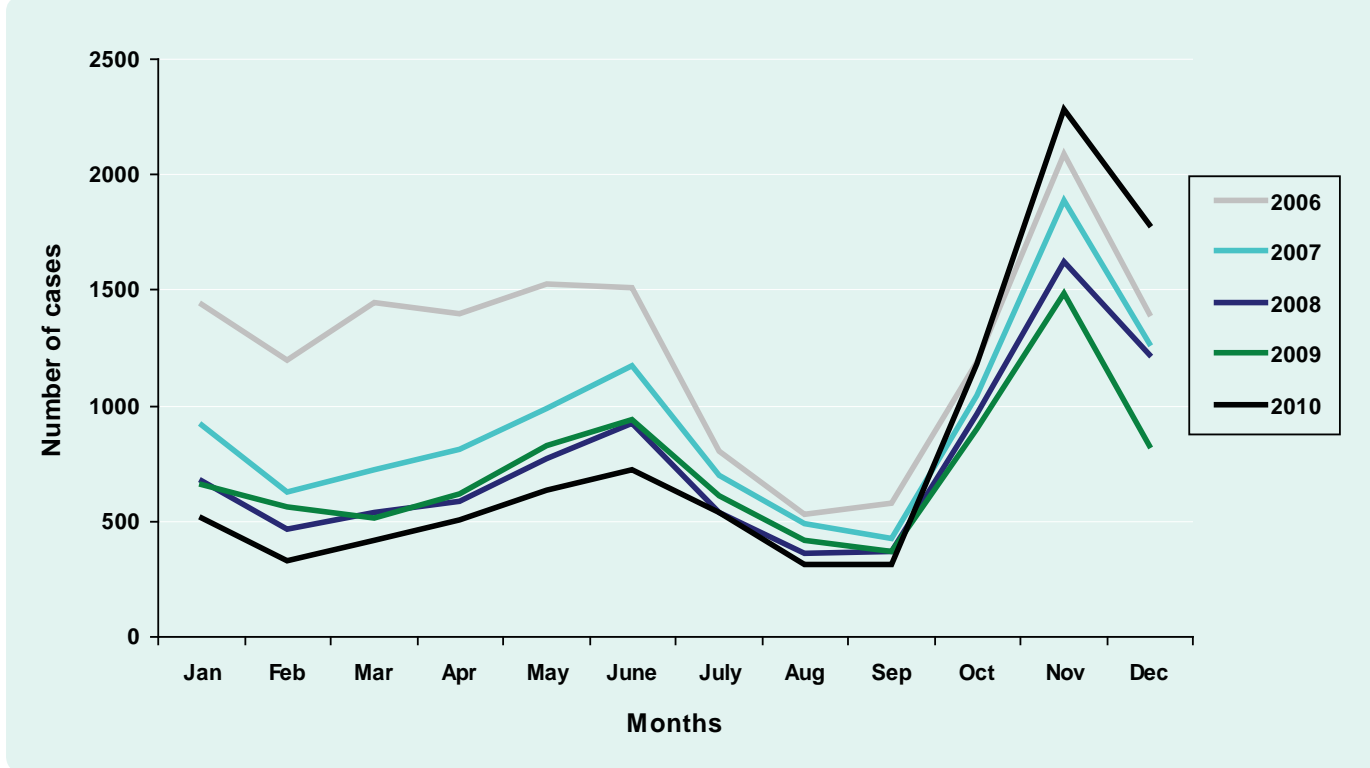

differential in gender distribution was similar among all five years examined. The male-to-female ratio of HFRS cases was similar in the cases reported by provinces (data not shown).

When cases were evaluated by employment status, it was determined that the majority of cases from 2006 to 2010 were represented by farmers $(66.83 \%$, Table 1). The employment type of HFRS cases was similar in the cases reported by provinces (data not shown).

\section{DISCUSSION}

Our data showed that the number of reported HFRS cases has declined remarkably after peaking in 1986. By 2009, the annual reported cases fell below 10000 . The changing trend of HFRS cases number during these years is consistent with other studies. ${ }^{9,14}$ However, some HFRS high risk areas still exist ${ }^{2}$ and have occasionally experienced an increase in cases in recent years. From 2006 to 2009, the downward trend slowed, while 
Figure 5. Haemorrhagic fever with renal syndrome age distribution, China, $2006-2010$

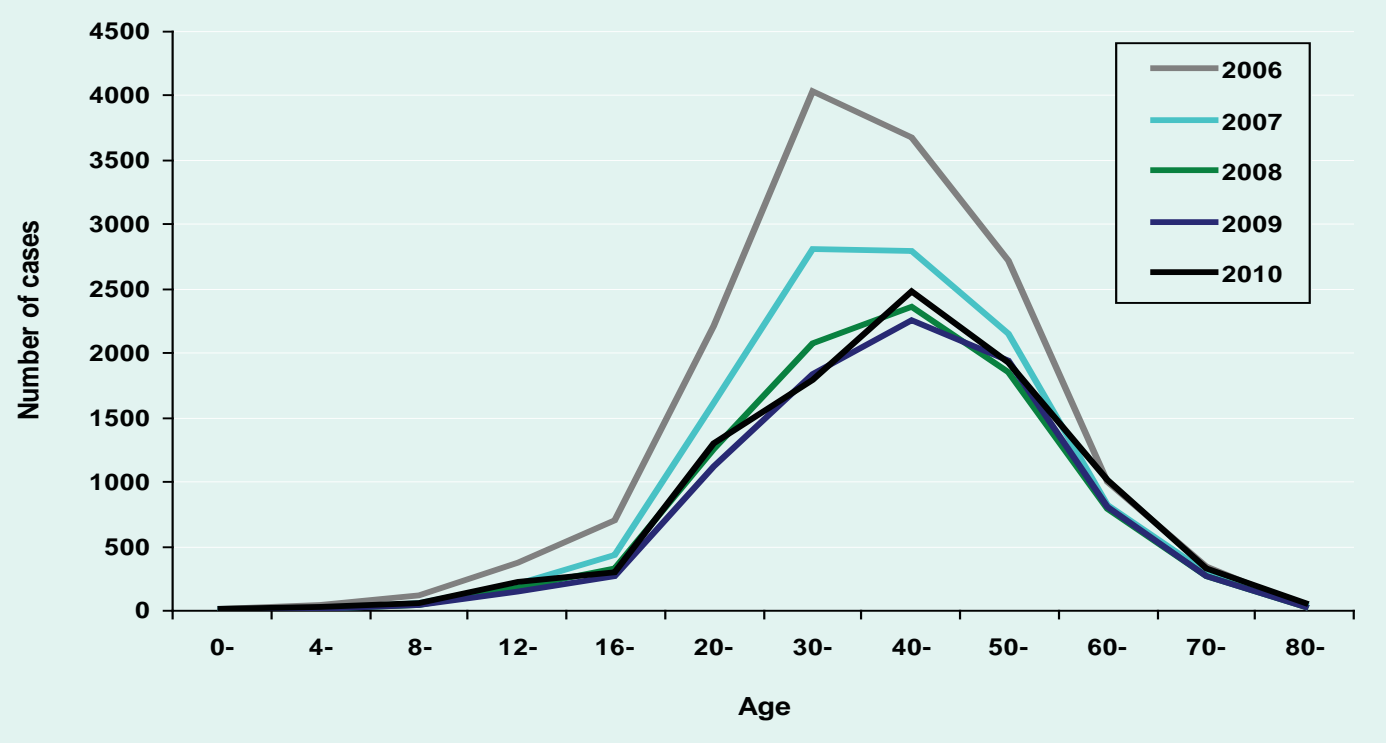

Table 1. Haemorrhagic fever with renal syndrome cases by employment status and sex, China, 2006-2010

\begin{tabular}{lrrrrrr}
\hline \multicolumn{1}{c}{ Employment status } & \multicolumn{2}{c}{ Number of cases (\%) } & \multicolumn{2}{c}{ Males (\%) } & \multicolumn{2}{c}{ Females (\%) } \\
\hline Farmers & 35732 & $(66.83)$ & 26840 & $(75.11)$ & 8892 & $(24.89)$ \\
Industrial labour or manual workers & 3914 & $(7.32)$ & 3442 & $(87.94)$ & 472 & $(12.06)$ \\
Household workers and unemployed & 2811 & $(5.26)$ & 1509 & $(53.68)$ & 1302 & $(46.32)$ \\
Students & 2465 & $(4.61)$ & 1870 & $(75.86)$ & 595 & $(24.14)$ \\
Migrant workers & 1905 & $(3.56)$ & 1677 & $(88.03)$ & 228 & $(11.97)$ \\
Functionaries & 1161 & $(2.17)$ & 1009 & $(86.91)$ & 152 & $(13.09)$ \\
Retirees & 1016 & $(1.90$ & 759 & $(74.70)$ & 257 & $(25.30)$ \\
Business service sector & 538 & $(1.01)$ & 423 & $(78.62)$ & 115 & $(21.38)$ \\
Teachers & 452 & $(0.85)$ & 344 & $(76.11)$ & 108 & $(23.89)$ \\
Catering food industry workers & 308 & $(0.58)$ & 251 & $(81.49)$ & 57 & $(18.51)$ \\
Herdsman & 141 & $(0.26)$ & 116 & $(82.27)$ & 25 & $(17.73)$ \\
Medical workers & 133 & $(0.25)$ & 101 & $(75.94)$ & 32 & $(24.06)$ \\
Preschool children & 131 & $(0.24)$ & 83 & $(63.36)$ & 48 & $(36.64)$ \\
Seafarers and long-distance drivers & 66 & $(0.12)$ & 66 & $(100.00)$ & 0 & $(0.00)$ \\
Fishermen & 59 & $(0.11)$ & 54 & $(91.53)$ & 5 & $(8.47)$ \\
Public service workers & 25 & $(0.05)$ & 14 & $(56.00)$ & 11 & $(44.00)$ \\
Nurses and nannies & 4 & $(0.007)$ & 1 & $(25.00)$ & 3 & $(75.00)$ \\
Others & 2028 & $(3.79)$ & 1685 & $(83.09)$ & 343 & $(16.91)$ \\
Unknown & 582 & $(1.09)$ & 455 & $(78.18)$ & 127 & $(21.82)$ \\
Total & 53471 & & 40699 & $(76.11)$ & 12771 & $(23.89)$ \\
\hline
\end{tabular}

the reported cases in 2010 were slightly higher than 2008 and 2009. This may be related to the outbreak in Shaanxi province in 2010 when HFRS cases were about twice that of 2009. Of those eight regions with a high incidence between 2006 and 2010, Heilongjiang, Jilin, Liaoning, Shandong, Shaanxi and Hebei had previously been identified high incidence regions in 2004 and $2005 .{ }^{14}$ A good example of the decline in cases is Hebei province, which was once considered among the high-incidence provinces, but it experienced a significant decrease in cases in 2007 (<400; a 64.99\% decrease from 2006), and has remained low. In 2010, Hebei was ranked as the $12^{\text {th }}$ most endemic HFRS region in China, as compared to $5^{\text {th }}$ in 2006. In contrast, Shaanxi represents the most intriguing and alarming region since it reported significantly higher numbers of HFRS cases in 2010. 
HFRS seasonality in China is characterized by a bimodal distribution pattern throughout the year. The spring peak often lasts for about three months from March to May. The winter peak is relatively rapid and short-lasting. The reasons for the peak occurring in winter and in spring may be related to several factors: ${ }^{15-20}$ viral types, hosts' reproduction and activities and natural or social factors e.g. flooding.

As in previous years, the late adolescent-to-adult age group accounted for the most cases of HFRS reported in the years from 2006 to 2010. Since 2008, seven of the particularly affected provinces (including five of the highest epidemic provinces and Hebei and Zhejiang provinces) have conducted immunization through the Expanded Programmes on Immunization (EPI) targeting the high-risk age group of 16-60 year olds; another 10 provinces were included since 2009. We noticed that the overall number of cases in this broad age group did decrease annually, and the decrease occurred for the entire study period both before and after the introduction of EPI, indicating that other factors may have contributed to the decline in cases.

The occupational and sex distribution in our study was consistent with the findings of an analysis of HFRS cases from 2004 and 2005. ${ }^{14}$ Young males, especially farmers, still are the focus for prevention and control. Since this is a rodent-borne infection, farmers are more likely to spend significant amounts of time in rodent-infested areas (barns, fields). In China, a farmer's living and working environment can lead to more exposure to rodents' feces, urine and saliva. In all occupations, except nurses and nannies, the number of male cases was more than the number of female cases. However, in other studies the male-to-female ratios of HFRS cases varies for the different species of virus. ${ }^{15,21}$ The different male-to-female ratio by virus type may be related to the different hosts of HFRS.

Unfortunately, we could not analyse the epidemiological characteristics of different viral types without the serum data of HFRS cases. Another limitation of the reported data is that with the development of monitoring and diagnostic technology, the detection of HFRS cases may be more accurate than that in the past. Moreover, with a deeper focus on HFRS by the Government, we saw that the laboratory-confirmed cases increased in 2010. The consistency of the surveillance data should be evaluated regularly.
Integrated intervention measures involving rodent control, environment management and vaccination have been implemented and may have played an important role in controlling HFRS in China. China still experiences significantly higher numbers of HFRS cases than other countries worldwide and an increase in cases was experienced in some areas in 2010. Our study has shown the recent epidemiologic characteristics of HFRS including regional, seasonal and population distribution.

\section{Conflicts of interest}

None declared.

\section{Funding}

The study was supported by a grant from the China Mega-Project for Infectious Diseases (2008ZX10004008).

\section{References:}

1. Fang $L Q$ et al. Spatiotemporal Trends and Climatic Factors of Hemorrhagic Fever with Renal Syndrome Epidemic in Shandong Province, China. PLoS Neglected Tropical Diseases, 2010, 4:e789. doi:10.1371/journal.pntd.0000789 pmid:20706629

2. Fang $L Q$ et al. Spatial analysis of hemorrhagic fever with renal syndrome in China. BMC Infectious Diseases, 2006, 6:77. doi:10.1186/1471-2334-6-77 pmid:16638156

3. Fang $L Q$ et al. [Using geographic information system to study on the association between epidemic areas and main animal hosts of hemorrhagic fever with renal syndrome in China] [in Chinese]. Chinese Journal of Epidemiology, 2004, 25:929-933. pmid: 15769318

4. Chen HX et al. HFRS surveillance in China. Zhonghua Liu Xing Bing Xue Za Zhi, 2002, 23:63-66.

5. Wu GH. [Study on transmission routes of EHF in recent years] [in Chinese]. Chinese Journal of Insect \& Equipment, 2008, 14:242-245.

6. Yang ZQ. The research progress of HFRS transmission routes. Practical Journal of Medicine \& Pharmary, 2005, 22:69-71.

7. Lee PW et al. Propagation of Korean hemorrhagic fever virus in laboratory rats. Infection and Immunity, 1981, 31:334-338. pmid:6111538

8. Hang CS et al. A clinical study on HFRS vaccine. Chinese Journal of Experimental and Clinical Virology, 2006, 20:423-425.

9. Zhang YZ, Xiao DL, Wang $Y$, et al. The epidemic characteristics and preventive measures of hemorrhagic fever with syndromes in China. Zhonghua Liu Xing Bing Xue Za Zhi, 2004, 25: 466-469. pmid: 15231118

10. Zhang GO, et al. Long-term epidemiological effect of hemorrhagic fever with renal syndrome vaccine in a large scale population. Journal of Shandong university (health sciences), 2007,45(10):981-984,991.

11. Wang $Q$ et al. [Epidemiology and surveillance programs on hemorrhagic fever with renal syndrome in Mainland China, 2005- 
2008] [in Chinese]. Chinese Journal of Epidemiology, 2010, 31: 675-680. pmid:21163102

12. Ministry of Health. Handbook of Epidemic Hemorrhagic Fever Prevention and Control. Beijing: China People's Health Publishing House, 1998.

13. Monitoring Program for Hemorrhagic Fever with Renal Syndrome. Beijing, Ministry of Health of the People's Republic of China, 2005 (in Chinese).

14. Wang XF et al. Epidemiological analysis of hemorrhagic fever with renal syndrome in China from 2004 to 2005. Disease Surveillance, 2007, 22:307-309.

15. Song G. [Achievements of EHF Prevention and Control in New China] [in Chinese]. Chinese Journal of Epidemiology, 2000, 21:378-382.

16. Zhang $Y Z$ et al. Hantaviruses in rodents and humans, Inner Mongolia Autonomous Region, China. Emerging Infectious Diseases, 2009, 15(6):885-891. doi:10.3201/eid1506.081126 pmid:19523286
17. Chen HX et al. Epidemiological studies on hemorrhagic fever with renal syndrome in China. Journal of Infectious Diseases, 1986, 154: 394-398. doi:10.1093/infdis/154.3.394 pmid: 19523286

18. Kim YS et al. Hemorrhagic fever with renal syndrome caused by the Seoul virus. Nephron, 1995, 71:419-427. doi:10.1159/000188762 pmid:8587622

19. Chen HX, Qiu FX. Epidemiological surveillance on the hemorrhagic fever with renal syndrome in China. Chinese Medical Journal, 1993, 106:857-863. pmid:7908258

20. Chen $\mathrm{HX}$ et al. [Characteristics of the distribution pf epidemic season of hemorrhagic fever with renal syndrome in different regions and different years in China] [in Chinese]. Chinese Journal of Experimental and Clinical Virology, 1994, 8:197-203.

21. Yan YC. LI Y, LV DX. [Epidemiological analysis of hemorrahgic fever with renal syndrome and control status] [in Chinese]. China Tropical Medicine, 2008, 8:465-467. 